\title{
Recognition Memory Shielded from Semantic but not Perceptual Interference in Normal Aging
}

\author{
D. Merika Wilson ${ }^{1, *}$, Kevin Potter ${ }^{1}$, Rosemary A. Cowell ${ }^{1, *}$ \\ Department of Psychological and Brain Sciences, University of Massachusetts Amherst
}

1. Department of Psychological and Brain Sciences

University of Massachusetts

Tobin Hall

135 Hicks Way

Amherst, MA 01003

*Corresponding authors:

D. Merika Wilson, dmerikawilso@umass.edu

Rosemary A. Cowell, rcowell@umass.edu 


\begin{abstract}
Normal aging impairs long-term declarative memory, and evidence suggests that this impairment may be driven partly by structural or functional changes in the medial temporal lobe (MTL). Theories of MTL memory function therefore make predictions for age-related memory loss. One theory - the Representational-Hierarchical account - makes two specific predictions. First, recognition memory in older participants should be impaired by feature-level interference, in which studied items contain many shared perceptual features such that those features appear repeatedly. Second, if the interference in a recognition memory task - i.e., the information that repeats across items - resides at a higher level of complexity than simple perceptual features, such as semantic gist, older adults should be less impacted by such interference than young adults. We tested these predictions using the Deese-Roediger-McDermott paradigm, by creating feature-level (i.e., perceptual) interference with phonemically/orthographically related word categories, and higher-level associative interference with semantically related word categories. Importantly, we manipulated category size in order to compare the effect of less versus more interference (i.e., small versus large category size), which served to (1) avoid potential item confounds arising from systematic differences between words belonging to perceptually- versus semantically-related categories, and (2) ensure that any effect of interference was due to information encoded at study, rather than pre-experimentally. Further, we used signal detection theory to interpret our data, rather than examining false alarm rates in isolation, thereby avoiding potentially confounding contamination of the memory measure by changes in response bias across conditions or groups. Older participants, relative to young adults, were relatively more impaired by perceptual interference and less impaired by semantic interference. This pattern seems at odds with many current theories of age-related memory loss, but is in line with the Representational-Hierarchical account.
\end{abstract}

Keywords: age-related memory deficits; Deese-Roediger-McDermott paradigm; signal detection theory; recognition memory 


\section{Introduction}

Long-term declarative memory function declines in old age. Deficits in older adults have been reported for several forms of long-term declarative memory including episodic, semantic, and spatial memory (Bäckman \& Nilsson, 1996; Newman \& Kaszniak, 2000; Rönnlund, Nyberg, Bäckman, \& Nilsson, 2005; Schaie, 2005). In particular, age-related impairments are frequently found in tasks that require the retrieval of information via associations, such as in contextual, associative, and source memory tasks (Chalfonte \& Johnson, 1996; Duarte, Henson, \& Graham, 2008; Naveh-Benjamin, 2000; Schacter, Kaszniak, Kihlstrom, \& Valdiserri, 1991; Schacter, Osowiecki, Kaszniak, Kihlstrom, \& Valdiserri, 1994; Silver, Goodman, \& Bilker, 2012; Spencer \& Raz, 1995; but see Campbell, Hasher, \& Thomas, 2010). Regarding the neuroanatomical changes associated with age-related memory decline, research has revealed disproportionate deterioration of medial temporal lobe (MTL) structures (Devitt \& Schacter, 2016; Raz, Gunning-Dixon, Head, Dupuis, \& Acker, 1998; Raz, Rodrigue, Head, Kennedy, \& Acker, 2004; Wang et al., 2002). Given the cognitive effects of aging, this finding fits with the well-documented role of the MTL in long-term declarative memory (Scoville \& Milner, 1957; Squire \& Wixted, 2011; Squire \& Zola-Morgan, 1991) and, more specifically, in associative or relational memory (e.g., Cohen et al., 1999; Eichenbaum, Otto, \& Cohen, 1994; Giovanello, Schnyer, \& Verfaellie, 2004).

One theory of the role of MTL structures in cognition - the Representational-Hierarchical (R-H) account (Bussey \& Saksida, 2002; Cowell, Bussey, \& Saksida, 2006; Sadil \& Cowell, 2017) has begun to amass empirical support for its explanation of mnemonic and perceptual deficits following brain damage (Barense et al., 2005; Barense, Gaffan, \& Graham, 2007; Barense, Groen, et al., 2012; Bartko, Cowell, Winters, Bussey, \& Saksida, 2010; Bartko, Winters, Cowell, Saksida, \& Bussey, 2007a, 2007b; Lee, Buckley, et al., 2005; Lee, Bussey, et al., 2005; Lee, Levi, Davies, Hodges, \& Graham, 2007; McTighe, Cowell, Winters, Bussey, \& Saksida, 2010). However, to our knowledge this theory has not been applied to the effects of aging on memory (but see Scheerer \& Marrone, 2014). And yet it makes clear predictions that distinguish it from other theories of age-related memory loss (e.g., Brainerd, Reyna, \& Howe, 2009; Naveh-Benjamin, 2000; Yassa \& Stark, 2011). In this article, we outline those predictions and report an empirical study that tested them.

Briefly, the R-H account assumes that the ventral visual pathway contains a hierarchically organized system of representations that culminates in the MTL. At the start of the pathway, in visual cortex, the simple features of visual stimuli (e.g., color, orientation) are represented individually, and those features are brought together into cohesive conjunctions of increasing complexity in progressively more anterior regions. MTL structures contain conjunctive 
representations of whole stimuli such as objects, scenes and episodic events. These representations determine the role of MTL structures in cognition: whenever a cognitive task requires discrimination between stimuli or events containing shared features, conjunctive MTL representations are needed to resolve the feature ambiguity (Bussey \& Saksida, 2002; Bussey, Saksida, \& Murray, 2002). This prediction has been confirmed using tasks that are traditional tests of memory (e.g., Bartko et al., 2010, 2007b; McTighe et al., 2010; Yeung, Ryan, Cowell, \& Barense, 2013) as well as tasks that tap perception (e.g., Barense et al., 2005; Barense, Groen, et al., 2012; Barense, Ngo, Hung, \& Peterson, 2012; Bartko et al., 2007a, 2007b; Lee, 2006; Lee, Bussey, et al., 2005; Lee, Bandelow, Schwarzbauer, Henson, \& Graham, 2006; Lee, Scahill, \& Graham, 2008), lending support to the proposal that the role of MTL structures in cognition is determined by representational content, rather than by specialization for a particular cognitive process.

If normal aging compromises MTL structures and the representations contained therein, then the R-H account makes predictions for age-related memory loss. That is, older adults provide a novel population in which to test the account, which predicts impairments in aging like those seen in patients with MTL damage, albeit to a lesser extent. One prediction of the R-H account for memory performance concerns 'feature-level interference' - a kind of perceptual interference in which features possessed by the to-be-discriminated test stimuli appear repeatedly during a task. According to the R-H account, such interference should impair the discrimination of old and new items when there is damage to conjunctive representations in MTL, because these representations are necessary for uniquely specifying an object (or event) and distinguishing it from similar items with shared features (Cowell et al., 2006). In support of this, studies have shown that feature-level interference causes impairments in recognition memory in individuals with MTL damage (Bartko et al., 2010, 2007b; McTighe et al., 2010; Yeung et al., 2013). Applying the R-H account to aging predicts the same effect in older relative to younger adults. However, since this prediction derives from the claim that conjunctive representations in MTL are compromised, a second, more counterintuitive prediction for interference also follows from the $\mathrm{R}-\mathrm{H}$ account. Consider a scenario in which the repeatedly appearing (i.e., interfering) attributes of the to-be-discriminated stimuli reside at the level of complex conjunctions, or high-level associations - effectively creating 'conjunction-level' interference. In this case, older adults should be paradoxically shielded from interference, because they are less able to encode that interference than young adults.

We operationalized these predictions for healthy older adults using a version of the DeeseRoediger-McDermott (DRM) recognition memory paradigm (Deese, 1959; Roediger III \& McDermott, 1995). In this paradigm, participants are presented with categories of related items at 
study, then tested with both studied items and unstudied lures, with some of the lures related in theme to the studied items. Participants of all ages tend to endorse related lures as studied with a higher probability than unrelated lures, a 'false memory' effect that could be explained in terms of interference between similar items (see Gallo, 2006 for a review). Our goal was to use this paradigm to create both feature-level and conjunction-level interference.

We used word stimuli and an experimental design that capitalized on the assumption that humans possess two separate representational hierarchies for words - one stream for phonemic/orthographic features and the other for semantic content. (This assumption is justified by considering that two very orthographically similar words such as TIDE and TILE have extremely dissimilar and separable semantic content; in addition, it is a central assumption of popular dualroute models of dyslexia and models of language production (Coltheart, Rastle, Perry, Langdon, \& Ziegler, 2001; Dell, 1986)). We propose that the phonemic/ orthographic hierarchy culminates in conjunctive representations of the combinations of phonemic/orthographic features that uniquely define whole words. In contrast, the semantic hierarchy culminates at the level of semantic themes or 'gist', which is represented via the semantic associations between individual words, rather than being contained within the individual word representations themselves. We further suggest that older adults have compromised representations at the top level of both hierarchies: aging renders whole-word level representations of the conjunctions of phonemic/orthographic features less precise, and renders representations of the semantic associations between words less robust. The notion that the MTL and nearby temporal cortex are involved in representing individual words phonemically/orthographically, particularly when those words are similar, and that aging can affect these representations, was inspired by a number of empirically-based claims: that perirhinal cortex is situated at the top of the visual word processing hierarchy (Liuzii et al., 2015); that perirhinal cortex is engaged specifically when abstract visual stimuli share a high degree of feature-overlap (Barense, Groen, et al., 2012; Barense, Rogers, Bussey, Saksida, \& Graham, 2010); and that older adults suffer increased interference, or competition, between phonological neighbors (Abrams, Trunk, \& Merrill, 2007; Gordon \& Kurczek, 2013). ${ }^{1}$ The notion that semantic properties and semantic associations depend upon MTL structures is supported by a considerable body of research

\footnotetext{
${ }^{1}$ We acknowledge the evidence for a role of the 'visual word form area' (VWFA, in the fusiform gyrus) in the representation of orthographic properties of words (e.g., Cohen \& Dehaene, 2004; Glezer, Kim, Rule, Jiang, \& Riesenhuber, 2015) and for a role of auditory cortex in the representation of phonemic properties (see Hickok \& Poeppel, 2007 for a review). We therefore remain cautious about tying the word-level of the phonemic/orthographic hierarchy to a single cortical location, or strictly to anterior temporal regions, but we suggest that it is nonetheless plausible to assume aging causes incipient damage to these conjunctive phonemic/orthographic representations.
} 
(Barense et al., 2010; Bruffaerts et al., 2013; Chadwick et al., 2016; Clarke \& Tyler, 2014; Manns, Hopkins, \& Squire, 2003; Warrington \& Shallice, 1984).

By using the DRM paradigm to create interference that affects these two putative processing streams in different ways, we created the conditions of feature-level and conjunction-level interference necessary for testing our predictions. Specifically, we created categories of items possessing shared phonemic/orthographic features but few semantic associations (e.g., DATE, FACE, FADE, FAKE, FAME, GATE, HATE, LATE, MATE, RATE), and categories of items that shared many semantic features but few phonemic-orthographic features (e.g., BABBLE, CRADLE, DIAPERS, HIGHCHAIR, LULLABY, PACIFIER, RATTLE, STORK, STROLLER, TEETHING). In the phonemic/orthographic categories, the shared information across items that provides the source of interference resides at the sub-word level: the letter- and phoneme- components appear repeatedly. In semantic categories, the shared information across items resides at the supra-word level: inter-item semantic associations. Thus, the phonemic/orthographic categories provide a 'feature-level interference' condition in which older adults should be less able to resolve the interference than young adults, because age is proposed to compromise the conjunctive representations of phonemic/orthographic features that uniquely define similar words. In contrast, the semantic categories provide a 'conjunction-level interference' condition in which older adults should be paradoxically shielded from interference, because age is proposed to compromise interitem associative representations, leading to less effective encoding of the interfering information.

Despite a number of prior studies investigating age-related changes in semantic and perceptual memory using the DRM paradigm, these predictions have not yet been tested. Difficulty in drawing relevant inferences from the literature stems from two sources. First, some prior DRM studies of memory in older adults are subject to limitations in task design or data analysis that allow for non-mnemonic explanations of the data, either in terms of differences in response bias between groups and conditions, or in terms of item confounds. Second, perhaps because of the variety of task designs and analyses employed, findings from prior DRM studies comparing perceptual and semantic interference in older adults are conflicting: some have reported agerelated deficits for perceptual but not semantic interference (Ly, Murray, \& Yassa, 2013), others have observed greater deficits for semantic than perceptual (Koutstaal et al., 2003), and others still have found deficits for both (Budson, Sullivan, Daffner, \& Schacter, 2003; Pidgeon \& Morcom, 2014).

Thus, the goal of the present study was to test the predictions of a new theoretical approach to understanding age-related memory loss with an empirical study that avoids some of the 
confounds that complicate the interpretation of prior, related research. Specifically, we included three important features in our experimental design and analysis, as follows.

First, we used signal detection theory to extract a measure of discriminability, d'. Unlike false alarm (FA) rates, $d$ ' is a measure of memory performance that is unconfounded by possible differences in response bias between groups or between conditions. In particular, we were concerned about the possibility that participants are able to maintain different criteria for different categories, even when the presentation of items from all categories is interleaved at test. This is plausible under the assumption that participants notice the themes of different categories to different extents, and allow the detection (or not) of a theme at the time of test to modify their decision process. Alternatively, the simple presence of systematically differing word properties across semantic versus phonemic/orthographic categories (e.g., word frequency, discussed further below) might differentially affect response criteria for the two conditions. Moreover, the propensity to use distinct criteria for different categories might differ between young and older participants, such that any group differences in the hit or FA rate reflect differences in response bias rather than memory. To avoid such contamination, we report hit and FA rates for the sake of completeness only, and focus exclusively on the d' measure to draw conclusions about memory.

Second, similar to many prior DRM studies, we interleaved the presentation of items from all categories in both study and test phases. This served two main purposes: (1) it decreased the salience of the shared themes within each category, increasing the chance that older adults with putatively compromised semantic/associative representations would fail to apprehend those associations; and (2) it helped avoid any confounding effects of fatigue on performance for words drawn from different category sizes (category size was a critical manipulation in our experimental design, as discussed below). That is, if words from small and large sized categories are tested in a blocked format, poorer performance on larger-sized categories could be induced either by additional interference from the category or by fatigue from being tested on a longer test list. In addition, interleaved presentation may have reduced participants' tendencies to use different response criteria across different category types at test (Shiffrin, Huber, \& Marinelli, 1995), but we are doubtful that it can eliminate this tendency entirely, as noted above.

Third, and most importantly, we manipulated category size, using categories of 2 and 8 items at study, in order to provide a measure of mnemonic interference for each condition that is not subject to item confounds. In a task design comparing perceptual with semantic interference, the experimenter's goal would typically be to observe an effect of interference type, allowing the conclusion that participants are differentially susceptible to the two types of interference (i.e., the 
build-up of interfering information in memory during the study phase). However, if only one category size is employed (e.g., Ly et al., 2013), any effect of interference type might be equally well explained by differences in inherent properties of words in the two interference types, such as frequency, pre-experimental familiarity, word length, and so on. That is, because words cannot be counterbalanced across the two conditions, their inherent properties are not controlled for when only a single category size is used. Instead, if each condition includes two category sizes (across which inherent word properties remain constant, if the assignment of particular words to category sizes is counterbalanced across participants) and we examine the difference between d' for 2-item and 8-item categories, we can measure the influence of study-related interference on recognition performance for each condition, in the absence of item confounds. Thus, our key dependent variable was an interference measure - the difference between d' for 2-item and d' for 8-item categories - and our critical prediction was an interaction between age group and interference type for this measure: older adults, relative to young adults, should suffer greater interference for phonemic/orthographic than for semantic categories.

\section{$\underline{\text { 2. Methods }}$}

\subsection{Participants}

A total of 120 participants were recruited from the University of Massachusetts-Amherst and the local community, including 40 older adults and 80 young adults. One older adult was excluded before analysis because MATLAB quit unexpectedly during the study phase. A further seven (three older and four younger) subjects were excluded during analysis (see section 2.4.1. Signal Detection Model). Of the remaining participants, older participants were between the ages of 60 and 92 years old $(M=71.4, S D=7.4)$ and young adults were between the ages of 18 and $30(M=$ $20.7, S D=2.7$ ). All participants spoke English fluently; had normal or corrected-to-normal vision; and were in general good health with no history of psychiatric or neurological conditions. Participants gave written informed consent after being told about the procedures of the experiment. Undergraduate students were compensated one extra credit that could be applied to an undergraduate psychology class and non-students were compensated \$10 per hour of participation.

\subsection{Materials}

We adapted study materials from Shiffrin et al. (1995). Stimuli consisted of 25 categories that were composed of 10 exemplar words and one prototype word (see Appendices A, B, and C for the complete stimulus set). Fifteen of these categories contained words that were all semantically similar within the category and tended to be relatively long (5-14 letters) with relatively low natural language frequency. The other ten categories contained words that were either 
phonemically or orthographically related to the prototype word and were either three-letter or four-letter monosyllabic words with a high natural language frequency. In addition to the words within the semantic and phonemic/orthographic categories, there were 44 extra words that met the same criteria as the words in semantic categories (i.e., language frequency and word length), and 20 extra words that met the same criteria as the words in the phonemic/orthographic categories; these words were not exemplars to any of the prototypes (i.e., they were unrelated extra words) and were used as buffer or distracter words (see section 2.3. Procedures). No words were offensive, emotionally loaded, or otherwise provocative.

Clearly, the two category types (phonemic/orthographic and semantic) were not matched in word language frequencies nor in word length. Regarding word frequency, several prior studies have revealed no effect of word frequency on memory performance in DRM-like paradigms (Madigan \& Neuse, 2004; Roediger III, Watson, McDermott, \& Gallo, 2001; Sherman \& Jordan, 2011), while others have found an effect on false recognition limited to the cases when conscious recollection is involved (Dewhurst, Hitch, \& Barry, 1998; Kinoshita, 1995). Although the influence of word frequency on recognition memory thus remains unclear, this property does not represent a confound for our study because we manipulated category size. That is, our critical measure was one of memory interference - the d'2-d'8 difference score - rather than absolute levels of d', and any effects of word frequency on absolute levels of recognition memory can be presumed removed because word frequency is the same across the 2-item and 8-item categories within each category type. Regarding word length, it has been found to affect false recognition only when the length of critical lures differs from the length of within-category words (Madigan \& Neuse, 2004), which was not the case in this paradigm.

\subsection{Procedures}

The experiment consisted of two phases: a study phase with 120 words and a test phase with 144 words (see Figure 1). Both phases were completed on a desktop computer with a separate monitor or on a laptop computer. During the study phase, words were presented for three seconds each, with the list of 120 words comprising eight or two exemplars from each of the ten semantic and ten phonemic/orthographic categories (100 in total), plus ten extra semantic buffer words at the beginning of the study list and ten more at the end, to prevent recency and primacy effects for the items of interest (i.e., the 100 items drawn from the thematic categories). These buffer items were not presented during the test phase. The selection of ten semantic categories out of a total possible 15 semantic categories, for use in the study phase, was counterbalanced across participants. Additionally, we randomized for each participant: the assignment of which categories 
contributed eight or two exemplars (with the constraint that five semantic and five phonemic/orthographic contributed eight exemplars, with the remaining categories contributing two); the specific exemplar words presented; and the order of presentation of all words during the study phase (with the constraint that the semantic buffer words remained in the first ten and last ten list positions). Consequently, semantic and phonemic/orthographic stimuli were intermixed. All words were presented in a white font on a black background.

Once the study phase was completed, the test phase began without any delay. The test list contained, in a randomized order for each participant, twenty extra semantic distracter words, twenty extra phonemic/orthographic distracter words and, from each of the 20 study categories, two studied exemplar words (targets; counterbalanced across subjects), two non-studied exemplar words (related lures; counterbalanced across subjects), and one prototype word (critical lure). Again, semantic and phonemic/orthographic stimuli were intermixed. The four remaining extra semantic buffer words were used in four practice trials that were presented at the start of the test phase, before any trials containing study category words. Data from these trials were discarded. The other extra words inserted into the test phase (twenty semantic and twenty phonemic/orthographic) were included as distractors (unrelated lures) to measure false memory for words that did not belong to any category and had no interference. Test trials were self-paced, with the participant's response cueing the presentation of the next word.

2.3.1. Task. At the beginning of the experiment, participants were informed that some words presented during the study phase would be repeated during the test phase and that there would be a final recognition memory test. Additionally, participants were informed that many of the words they were to see would seem similar or related. During the study phase participants were asked to rate the pleasantness of the presented word by pressing keys numbered 1-5. On this 5 -point scale, 1 was considered very pleasant, 5 was very unpleasant, and 3 was neutral. In the test phase, participants were prompted to give a rating of their confidence that the current word was seen before. This confidence was measured on a 6-point scale where pressing 1 meant that he/she was very sure the item was a new word and pressing 6 meant that he/she was very sure it was an old word.

2.3.2. Neuropsychological Tests. After completion of the experimental study, all older adult participants completed an additional one-hour neuropsychological battery, to assess cognitive abilities and confirm healthy cognitive status. These tests assessed memory, thinking, language, and visual perception and included Wechsler Memory Scale-IV Logical Memory I \& II (Wechsler, 2009); Trails Making Test Parts A and B (Lezak, Howieson, \& Loring, 2004); Wechsler Adult Intelligence 
Scale-IV Digit Span (Wechsler, 2008); Mini-Mental State Examination (Folstein, Folstein, \& McHugh, 1975); and Visual Object Space Perception Silhouettes (Warrington \& James, 1991). Young adult participants did not complete the neuropsychological tests.

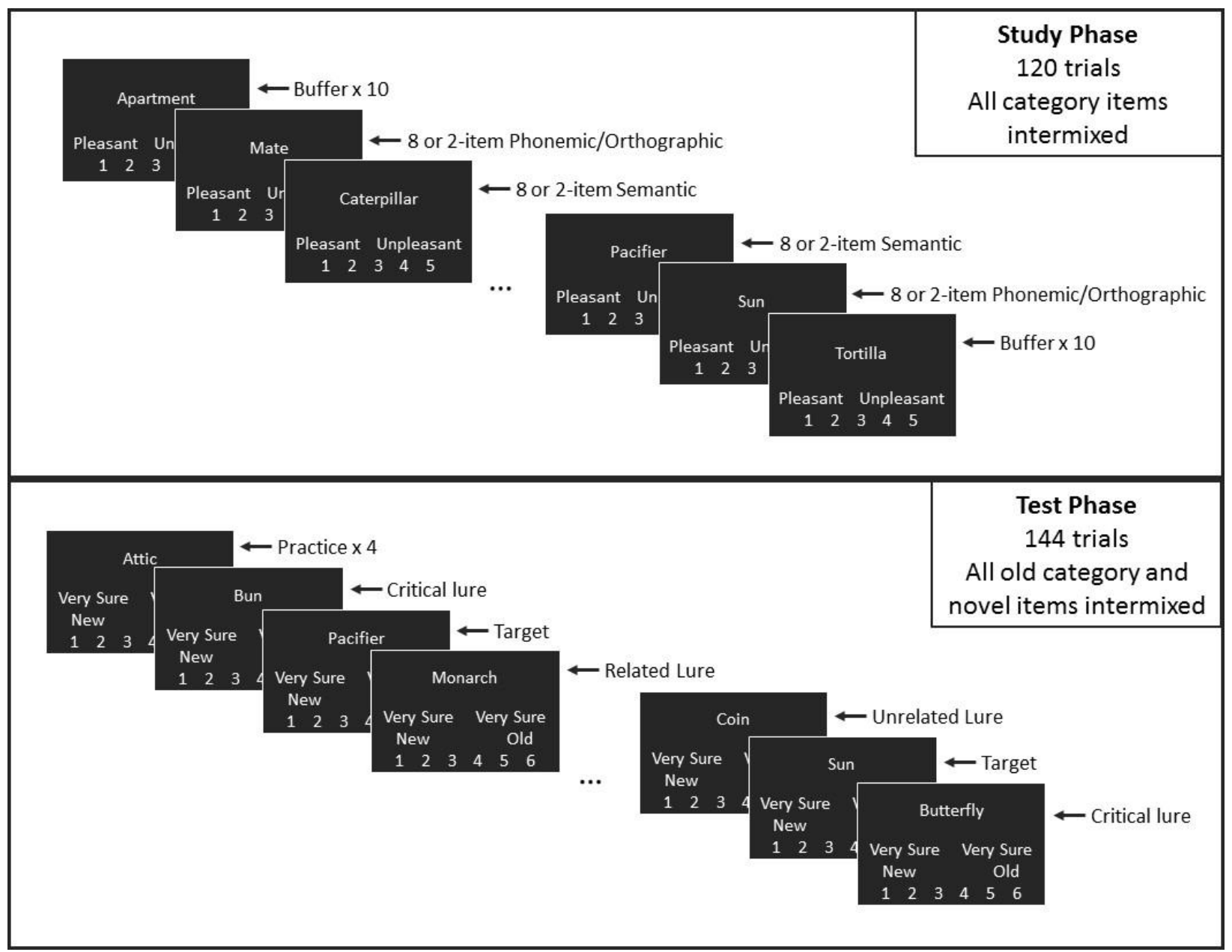

Figure 1: Illustration of Experimental Paradigm. The study phase comprised 120 trials, 20 of which served as "buffer" trials, 10 at the beginning and 10 at the end of the study list, to prevent recency and primacy effects. The remaining 100 study trials sampled words from 10 semantic categories and 10 phonemic/orthographic categories (drawing 2 items from 5 of the semantic categories and 8 items from the other 5 semantic categories; likewise for phonemic/orthographic categories). Words sampled from these categories were intermixed in the study phase and appeared as one long list. The test phase comprised 144 trials, including 4 practice trials at the beginning of the test list. Of the remaining 140 trials, sampled words were either targets (studied category exemplar words), critical lures (non-studied category prototype words), related lures (non-studied category exemplar words), or unrelated lures (non-studied words unrelated to any category). Words from these categories were intermixed in the test phase, appearing as one long list. During the study phase, participants were asked to respond during the $3000 \mathrm{~ms}$ presentation of each word; during the test phase, participants could take as long as they needed to respond. 


\subsection{Statistical Analysis}

2.4.1. Signal Detection Model. Signal detection theory (SDT) was used to analyze the data. SDT models assume that a participant's response is based on a combination of the degree of discriminability - in this context, the ability to detect whether a word was previously studied - and a criterion $(k)$ value, which governs the participant's decision rule (Macmillan \& Creelman, 2005). In this model, on any given trial a participant must make an old-new recognition decision based on a sampled 'memory strength' value for the current word. Memory strength values are assumed to vary from trial to trial according to a normal distribution (see Figure 2). We assumed differing distributions of memory strength for each type of word (e.g., target, lure, etc.). The likelihood of a particular response (e.g., a miss or a hit) is determined from the area under the curve to the left or right of the criterion value.

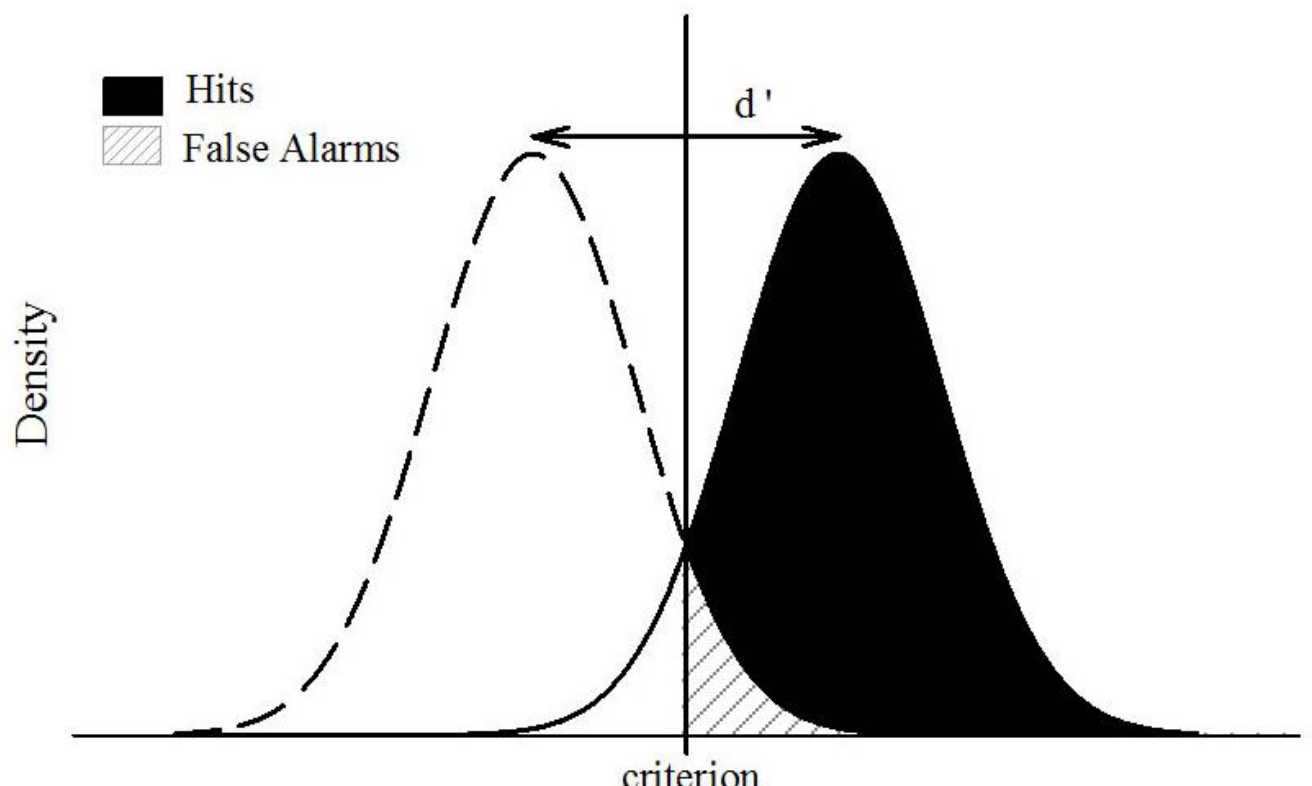

Familiarity Value

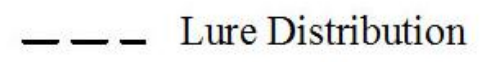

Target Distribution

Figure 2: Illustration of Signal Detection Theory Measurements. The discriminability measure $\mathrm{d}^{\prime}$ is calculated by converting the hit and false alarm rate to $\mathrm{z}$ scores and finding the difference, $d^{\prime}=z[$ Hits $]-z$ [False Alarms]. Hits are determined from the area under the target distribution to the right of the criterion (i.e., when the word is old and the participant's response is old). False alarms are determined from the area under the lure distribution to the right of the criterion (i.e., when the word is new and the participant's response is old). 
Typically, targets (i.e., previously seen words) have a greater mean memory strength than lures and so the memory strength distribution for targets is shifted to the right; however, there exist some items with ambiguous memory strength values, where the target and lure distributions overlap. Discriminability (d') is the difference between the mean memory strengths for the target and lure distributions, taking into account the variance of those distributions. A greater d' reflects less overlap between the two distributions. In psychological experiments, it is unlikely that the two distributions do not overlap at all (corresponding to a participant with perfect recognition performance).

In order to make a recognition decision, participants must employ a criterion, $k$. Any memory strength value above $k$ elicits an "old" response and any value below $k$ elicits a "new" response. This leads to four possible response types: 1) hits when the word is old and the response is old; 2) FAs when the word is new and the response is old; 3) misses when the word is old and the response is new; and 4) correct rejections when the word is new and the response is new. To calculate d', it is sufficient to know the frequencies of hits and FAs, along with total number of target and lure trials. The proportion of misses and correct rejections is simply one minus the proportion of hits and FAs, respectively. The predicted rates of hits and FAs can be calculated by the proportion of area under the target distribution curve to the right of $k$ and the area under a given lure distribution curve to the left of $k$, respectively. Therefore, $\mathrm{d}^{\prime}$ and $k$ provide a complete description of a participant's recognition performance, including their hits and FAs. Assuming $k$ is fixed to zero, a d' value of zero would produce $50 \%$ hits and FAs (i.e., chance performance). As d' increases, the percentage of hits increases, and the percentage of FAs decreases.

We applied a signal detection model to derive estimates of the discriminability between targets and lures for each condition, defined by lure type (critical, related), interference type (phonemic/orthographic, semantic), and category size (large, small). We did not examine the discriminability of targets from unrelated lures (which differed neither by interference type nor category size), since this was of no a priori theoretical interest. Discriminability, measured as d', was derived using the algebraic formula, $d^{\prime}=z$ [Hit rate $]-z$ [FA rate], separately for each participant in each condition. The six confidence ratings given by participants were collapsed into a dichotomous response (old/new, in which responses 1-3 were mapped to 'new' and responses 4-6 were mapped to 'old') because an insufficient number of participants used the full scale when responding (see Figure 3). Consequently, the model assumed variance equal to one. Therefore, a total of eight values were calculated for each participant: two d' values, namely d' between targets and critical lures and d' between targets and related lures, for each of the four possible 
combinations of category size (2-item or 8-item) and interference type (phonemic/orthographic or semantic).

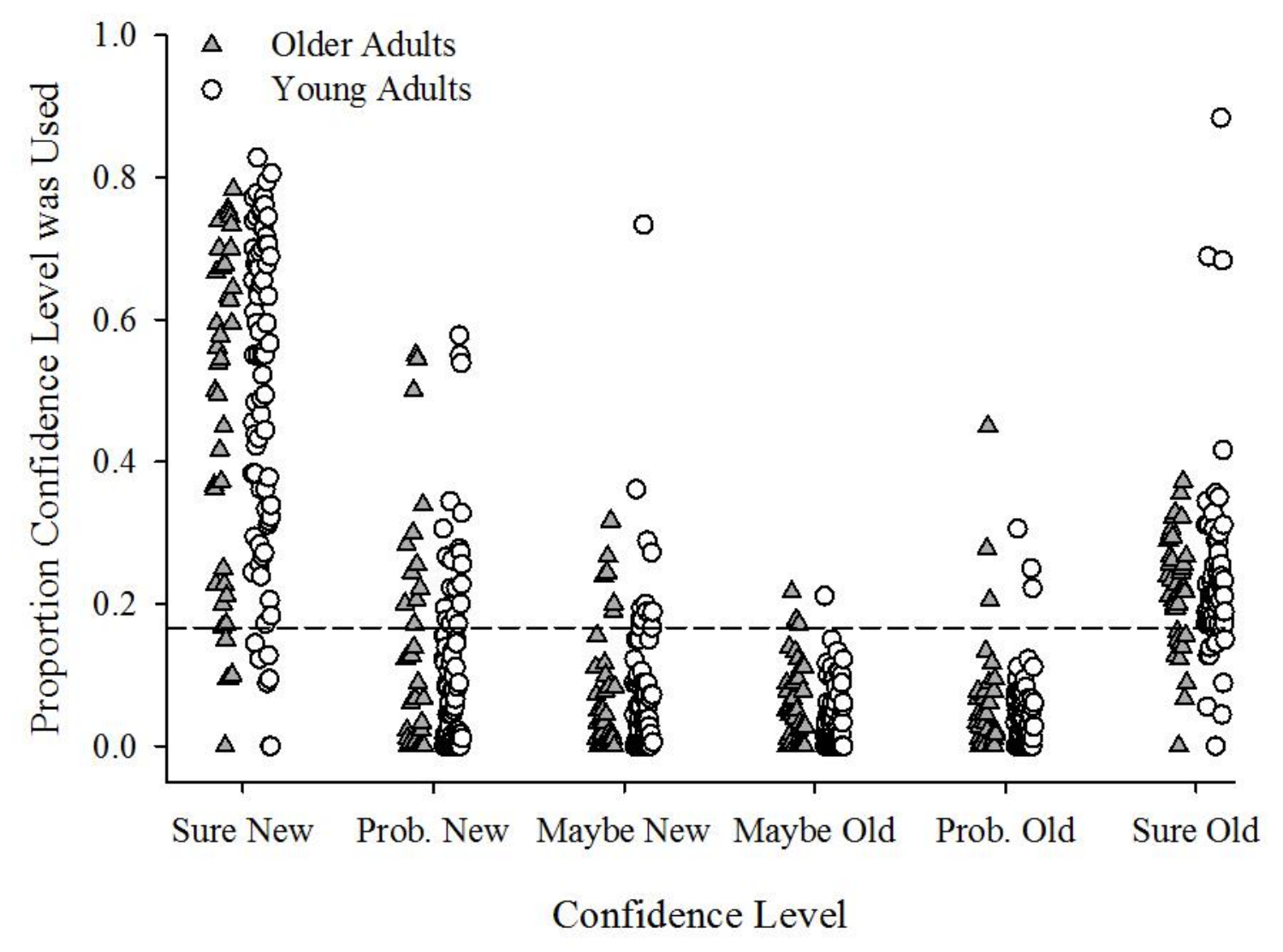

Figure 3: Proportion of Times a Confidence Level was used by Older and Young Subjects. Each point shows the proportion of responses for one subject averaged across conditions. The dotted line shows proportions corresponding to equal use of each level.

Before deriving the values of the signal detection model we pre-screened the data in three ways. First, we identified subjects whose average accuracy across conditions was significantly lower than chance $(0.41 ; n=4)$ and relabeled their old/new responses to the opposite mapping, assuming that these participants had accidentally switched the keys used for indicating 'old' and 'new' during the experiment. Second, any subject whose average accuracy fell within a 95\% confidence interval around chance performance (0.41-0.59) was removed from the analysis $(n=7)$. Third, we applied the Hautus (1995) correction for extreme proportions and their biasing effect on d', by adding . 5 to the response count in every cell (i.e., the total response count per condition per participant) and 1 to the total number of trials in each condition; this method has been found to 
produce less biased estimates of $d^{\prime}$ than the $1 /(2 \mathrm{~N})$ method that corrects only extreme proportion values of 0 or 1 (Snodgrass \& Corwin, 1988).

As discussed above, the category size condition was critical in providing an index of the extent to which any observed false memory for related lures is induced by mnemonic interference (i.e., by studying a category of related items) rather than by inherent properties of the words that are used to form related categories in the DRM paradigm. For example, there may be systematic differences between semantic and phonemic/orthographic words in terms of backward association strength, base frequency, or pre-experimental familiarity, making one class of lure more prone to be falsely remembered, or one class of target more difficult to remember after study. However, these inherent word properties should be the same regardless of category size (in this study, 2-item versus 8-item), provided that the assignment of items to category sizes is counterbalanced across participants. Therefore in order to control for the effects of inherent word properties we calculated the difference between the d' scores for 8-item and 2-item categories, for each participant within each interference type (phonemic/orthographic, semantic). This d' difference score (d'2 - d'8) not only addresses potential confounds caused by inherent word-category properties, but can also be considered a measure of study-related interference (i.e., the effect of seeing more related lures) on recognition memory performance.

In drawing conclusions from the data we focused primarily on the discriminability of targets from related lures, rather than targets from critical lures. This is because related lures have a key advantage over critical lures: related lures can be counterbalanced across participants, removing any effect of inherent word properties. In DRM word categories, the word that serves as the critical lure is fixed and (similar to potential differences between perceptual versus semantic category words) may have certain properties of frequency, pre-experimental familiarity, and backward association strength that differ from all other words in the category, making it more prone to be falsely remembered. Moreover, these distinctive properties of the critical lure may be more pronounced for one condition (semantic versus perceptual) than the other, complicating the interpretation of any condition effects. In contrast, related lures are drawn randomly from the unstudied members of a category, which allows counterbalancing across participants, thereby removing any potential item confounds caused by properties specific to critical lures.

\subsubsection{Statistical Analyses of Recognition Memory and Interference Measures. All} ANOVA's were calculated using the 'ez' R package (Version 4.2; Lawrence, 2015) and all $t$-tests were Welch's t-tests calculated using the base 'stats' R package (Version 3.2.4; R Core Team, 2016). First, discriminability (d') scores were analyzed in a four-way (2x2x2x2) ANOVA, with a between- 
subjects factor of Age (older and young adults); and within-subjects factors of (1) Interference Type (phonemic/orthographic and semantic); (2) Item Type (critical lures and related lures); and (3) Category Size (2-item and 8-item categories).

Next, we assessed the d' difference score (d'2 - d'8), to measure study-related interference. We analyzed d'-difference scores using a three-way (2x2x2) ANOVA, with factors Age Group, Interference Type and Item Type, as above. According to the R-H account, we expected older adults to be affected less by study-related interference for semantic words, but suffer from more interference for phonemic/orthographic words, relative to young adults. This prediction should manifest as an interaction between Age and Interference Type; specifically, older adults should have smaller d'2 - d'8 than young adults in the semantic condition, but a larger d'2 - d'8 in the phonemic/orthographic condition. The cleanest test of this prediction was provided by related lures, which, unlike critical lures, were counterbalanced to eliminate item confounds.

\section{$\underline{\text { 3. Results }}$}

\subsection{Neuropsychological Test Performance}

Results of the neuropsychological battery are shown in Table 1. Older adults demonstrated intact group performance on all cognitive tasks, with average performance within the normal range relative to established norms or within established passing cutoff scores (Crum, Anthony, Bassett, \& Folstein, 1993; Folstein et al., 1975; Tombaugh, 2004; Warrington \& James, 1991; Wechsler, 2008, 2009). Individually, all older participants included in the analysis passed the study's inclusion criterion of a score greater than 25 on the Mini-Mental State Examination.

\subsection{The effects of semantic and perceptual interference on recognition memory}

Hits and FAs for all participants in all conditions are shown in Table 2, separated according to Item Type (Unrelated Lures were not assigned to any category, and thus had no perceptual/semantic or 2-item/8-item status). Because these raw proportion scores are influenced by both memory and response bias and we are interested only in memory, we focused our statistical analyses on the d' measures derived from them, shown in Figures 4 and 5.

We began by analyzing discriminability ( $d^{\prime}$ ) of targets from critical and related lures. A fourway ANOVA with d' as the dependent variable revealed main effects of Age Group (old, young), $F$ (1, $110)=4.19, p=.043$, Interference Type (phonemic, semantic), $F(1,110)=11.44, p=.001$, Item Type (critical, related), $F(1,110)=161.2, p<.001$, and Category Size $(2$-item, 8 -item), $F(1,110)=$ 27.51, $p<.001$ (see Figure 4). Additionally, there were significant interactions between Age Group and Interference Type, $F(1,110)=4.52, p=.036$; Interference Type and Item Type, $F(1,110)=$ 5.91, $p=.012$; and Item Type and Category Size, $F(1,110)=19.8, p<.001$. Because related lures 
provided a cleaner test of our predictions than critical lures, and because we revealed a main effect of Item Type, we followed this analysis with two additional 2x2x2 ANOVA's examining critical lures and related lures separately.

Table 1: Older Adult Average Raw Scores for Neuropsychological Battery

\begin{tabular}{lc}
\multicolumn{1}{c}{ Test (Maximum score) } & M (SD) \\
\hline MMSE (/30) & $29.0(1.1)$ \\
WMS-IV LM Immediate Recall (/50) & $23.6(5.4)$ \\
WMS-IV LM Delayed Recall (/50, 20-min delay) & $19.6(6.3)$ \\
WMS-IV LM Recognition (/30) & $22.2(3.9)$ \\
Trails A & $24.0 \mathrm{~s}(6.3 \mathrm{~s})$ \\
Trails B & $64.2 \mathrm{~s}(37.2 \mathrm{~s})$ \\
WAIS-IV Digit Span Forward (/9) & $6.7(1.1)$ \\
WAIS-IV Digit Span Backward (/8) & $4.8(1.1)$ \\
VOSP Silhouettes (/30) & $19.4(4.9)$ \\
\hline
\end{tabular}

Note: The mean (M) performance on all cognitive tasks was within the normal range relative to established norms or within established passing cutoff scores. MMSE = Mini-Mental State Examination; WMS-IV LM = Wechsler Memory Scale, $4^{\text {th }}$ ed., Logical Memory subtest; WAIS-IV = Wechsler Adult Intelligence Scale, $4^{\text {th }}$ ed.; VOSP = Visual Object Space Perception battery.

For related lures, we found main effects of Interference Type, $F(1,110)=18.42, p<.001$, with semantic d' scores greater than phonemic/orthographic d' scores, and Category Size, $F(1,110)$ $=5.66, p=.019$, with 2-item d' scores greater than 8-item d' scores, as expected given the greater interference introduced by 8-item categories. There was a borderline interaction between Age Group and Interference Type, $F(1,110)=3.48, p=.065$, in which young adults showed a greater d' advantage for semantic over phonemic/orthographic categories than did older adults. Finally, there was a three-way interaction between Age Group, Interference Type, and Category Size, $F(1,110)=$ $4.76, p=.031$. For older adults, d' differed numerically less between 2 -item and 8-item categories for semantically related categories than for phonemically/orthographically related categories. In contrast, for young adults, d' differed numerically more between 2-item and 8-item categories for semantically related categories than for phonemically/orthographically related categories. Thus, d' for older adults tended to be more influenced by phonemic/orthographic interference than semantic, whereas for young adults the reverse was true (Figure 4). This three-way interaction in the d' scores is equivalent to a 2-way interaction between Age and Interference Type in the d' 
difference scores (i.e., d'2 - d'8), as predicted by the R-H account. Analyses of the d' difference (i.e., 'interference') scores are reported below.

\begin{tabular}{|c|c|c|c|c|c|c|c|c|c|c|c|c|c|}
\hline \multirow{4}{*}{$\begin{array}{l}\text { Category } \\
\text { Size }\end{array}$} & \multirow{2}{*}{\multicolumn{3}{|c|}{$\begin{array}{l}\text { True recognition } \\
\text { Targets }\end{array}$}} & & \multicolumn{8}{|c|}{ False recognition } & \multirow{4}{*}{$\underline{\text { Unrelated lures }}$} \\
\hline & & & & & \multicolumn{4}{|c|}{ Critical lures } & \multicolumn{4}{|c|}{ Related lures } & \\
\hline & \multicolumn{2}{|c|}{ Semantic } & \multicolumn{2}{|c|}{ Perceptual } & \multicolumn{2}{|c|}{$\underline{\text { Semantic }}$} & \multicolumn{2}{|c|}{ Perceptual } & \multicolumn{2}{|c|}{$\underline{\text { Semantic }}$} & \multicolumn{2}{|c|}{$\underline{\text { Perceptual }}$} & \\
\hline & 2 & 8 & 2 & 8 & 2 & 8 & 2 & 8 & 2 & 8 & 2 & 8 & \\
\hline $\begin{array}{l}\text { Younger } \\
\text { adults }\end{array}$ & & & & & & & & & & & & & \\
\hline Mean & .89 & .89 & .85 & .90 & .17 & .37 & .21 & .40 & .12 & .20 & .20 & .28 & .09 \\
\hline SEM & .014 & .018 & .017 & .017 & .023 & .031 & .026 & .027 & .013 & .017 & .021 & .023 & .009 \\
\hline $\begin{array}{l}\text { Older } \\
\text { adults }\end{array}$ & & & & & & & & & & & & & \\
\hline Mean & .76 & .81 & .76 & .76 & .15 & .39 & .17 & .32 & .11 & .18 & .12 & .19 & .06 \\
\hline SEM & .034 & .033 & .039 & .041 & .027 & .043 & .029 & .043 & .020 & .032 & .025 & .030 & .011 \\
\hline
\end{tabular}

For critical lures, there were main effects of Age Group, $F(1,110)=6.39, p=.013$, and Category Size, $F(1,110)=40.5, p<.001$. Younger adults' d' scores were greater than older adults' d' scores, and d' for 2-item categories was greater than d' for 8-item categories. There was also a trend towards an interaction between Age Group and Interference Type, $F(1,110)=3.08, p=.082$, in which - similar to the pattern seen in related lures - young adults showed a greater d' advantage for semantic over phonemic/orthographic categories than did older adults. In the critical lures d' scores, the three-way interaction between Age Group, Interference Type and Category Size was not significant.

Next, we analyzed the d'2 - d'8 interference score in a 3-way ANOVA with factors Item Type, Interference Type and Age Group. This was the most important analysis because, as explained above, this dependent variable minimizes the effect of item confounds by exploiting the category size manipulation. We found a main effect of Item Type (critical lures, related lures), $F(1,110)=$ $19.78, p<.001$ (Figure 5). To recap, d'2 - d'8 serves as a measure of study-related interference (i.e., the effect of seeing more related items) on recognition memory performance. Because the scores 
from critical and related lures differed significantly, and because we had a priori reasons to place greater confidence in the data from related lures, we analyzed them separately.

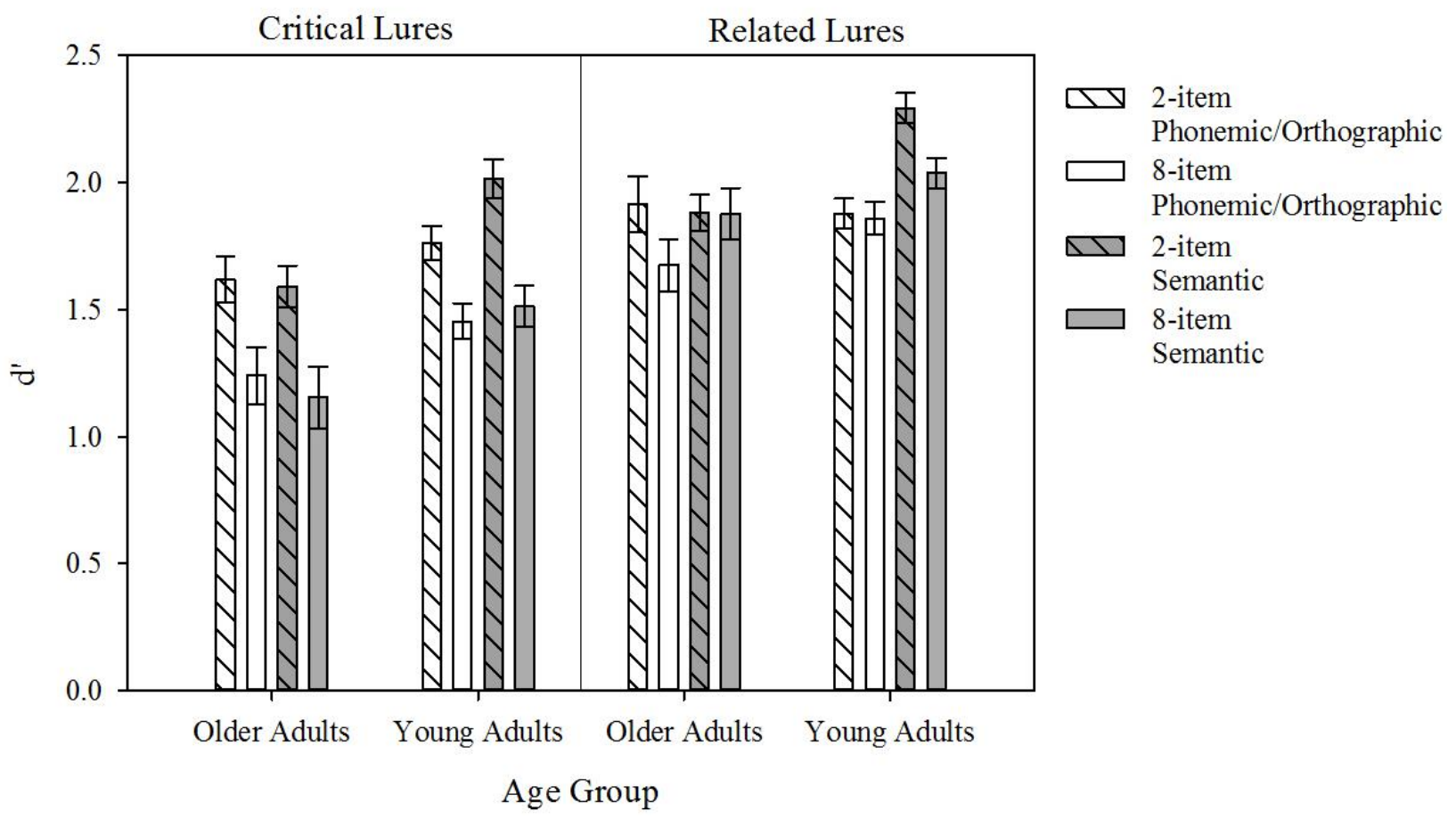

Figure 4: Analysis of d' between Target and Critical Lure or Target and Related Lure Distributions across Age Group, Category Size, and Interference Type. A main effect of Item Type $(p<.001)$ supported separate analyses for critical and related lures. For related lures, there was a three-way interaction between Age Group, Category Size and Interference Type $(p=.031)$, in which older adults' d' scores differed numerically more between 2-item and 8-item categories for phonemically/orthographically related categories than semantic categories, whereas the opposite pattern held for young adults. This interaction was not significant for critical lures. Error bars are within-subject standard error of the mean.

For d'2 - d'8 scores from related lures, we found the predicted 2-way interaction between Age Group and Interference Type, $F(1,110)=4.76, p=.031$. Numerically, older adults had greater d'2 - d'8 scores than young adults for phonemically/orthographically related categories, whereas young adults had greater d'2 - d'8 scores than older adults for semantically related categories. Within this interaction, there was no simple main effect of Age Group for either Interference Type. However, for semantically related categories, older adults' d'2 - d'8 scores did not differ significantly from zero, Welch's $t(35)=0.03, p=.976$, but young adults' scores did, $t(75)=2.91, p=$ .005 . That is, older adults were not impaired by an increase in semantic interference, but young adults were. Although these two results cannot be taken as evidence that young and older adults differed from each other within semantic categories, they are nonetheless instructive about the nature of the interaction between Age Group and Interference Type. In contrast, for 
phonemically/orthographically related categories, neither d'2 - d'8 scores from older adults, $t$ (35)

$=1.55, p=.13$, nor young adults, $t(75)=0.20, p=.84$, differed significantly from zero. Again,

although the simple main effect of Age Group was not significant, it is informative to note that the numerical pattern seen in phonemically/orthographically related categories was in the opposite direction from that seen in semantic categories, which presumably contributed to the overall interaction that was observed. For completeness, we also examined the complementary simple main effect - Interference Type within each Age Group separately - revealing no difference in older adults, and a borderline significant difference in young adults $(\mathrm{p}=0.057)$ in the direction of greater d'2-d'8 scores for semantic than phonemic/orthographic categories. In sum, the presence of a 2way interaction between Age and Interference Type for the d' difference scores provides evidence that - as predicted by the R-H account - recognition memory in older adults, relative to young adults, is less impaired by semantic interference and more impaired by phonemic/orthographic interference.

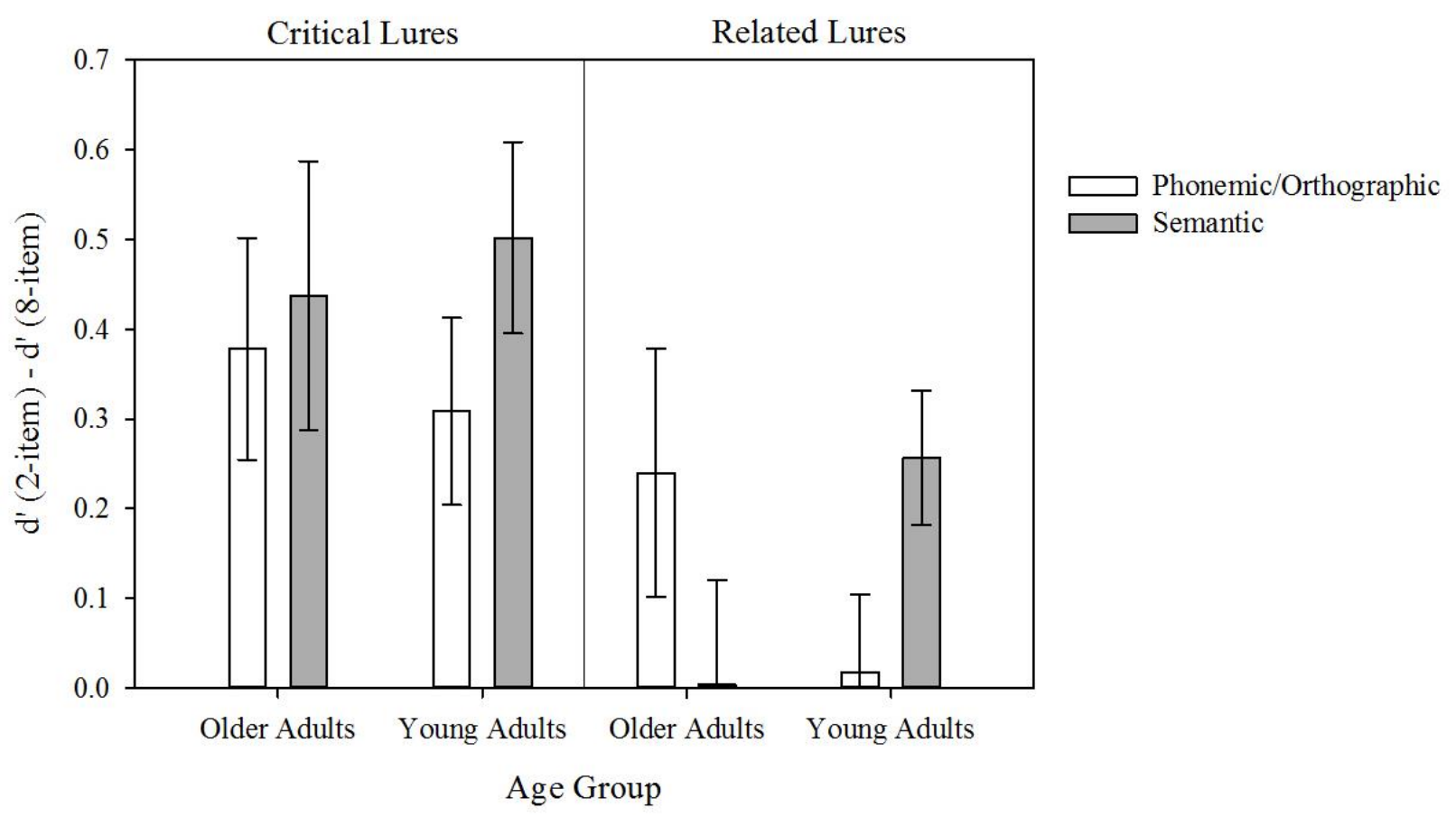

Figure 5: Analysis of Study-Related Interference on d' between Target and Critical Lure or Target and Related Lure Distributions across Age Group and Interference Type. Study-related interference was measured by subtracting the d' score from 8-item categories from the d' score from 2-item categories (d' [2-item] - d' [8-item]) within a given condition (Item Type, Age Group, Interference Type). For related lures, there was an interaction between Age Group and Interference Type ( $p=0.031)$, such that older adults' d' scores were impaired less by semantic and more by phonemic/orthographic interference compared to young adults. There were no significant effects for critical lures, though a similar numerical pattern can be seen. Error bars are within-subject standard error of the mean. 
For critical lures, there were no significant effects in the d'2 - d'8 scores, but general patterns similar to those observed in related lures were seen. That is, older adults produced numerically greater d' difference scores than young adults for phonemic/orthographic critical lures, while young adults gave numerically greater d' difference scores than older adults for semantic critical lures.

\section{Discussion}

The key result was a differential effect of perceptual and semantic interference on recognition memory in older compared with younger adults. Specifically, recognition memory in older adults was impaired relatively more by perceptual interference and less by semantic interference than was the case in young adults. This finding was predicted by the $\mathrm{R}-\mathrm{H}$ account of cognition, applied here for the first time to memory performance in aging.

Several theories have previously been put forward to explain age-related memory loss. One theory holds that aging causes deficits primarily in associative memory, or the ability to bind information together into a cohesive, complex unit (Chalfonte \& Johnson, 1996; Naveh-Benjamin, 2000; Naveh-Benjamin, Hussain, Guez, \& Bar-On, 2003). Other theorists have suggested that while older adults can encode and retrieve memory for the gist of an experience, mnemonic deficits emerge because verbatim traces for individual items are compromised (Fuzzy Trace Theory; Brainerd, Reyna, \& Howe, 2009). Another recent theory hypothesizes a central role for pattern separation in memory, and proposes that this function depends critically on the hippocampus, which is compromised by aging (Holden, Hoebel, Loftis, \& Gilbert, 2012; Schacter, Koutstaal, \& Norman, 1997; Stark, Yassa, \& Stark, 2010; Yassa et al., 2011). These three theories make different predictions for memory function in old age, including whether age-related impairments occur to different extents for specific classes of mnemonic content such as perceptual and semantic. The Associative-Deficit Hypothesis (Naveh-Benjamin, 2000) predicts that memory should be impaired by aging whenever the stimulus material is associative (Naveh-Benjamin et al., 2003); a corollary of this prediction is that memory should not be impaired by perceptual interference in which the interfering material is merely similar to, rather than associatively related to, studied material. In

contrast, Fuzzy Trace Theory predicts that old age should impair memory for any fine-grained details - whether perceptual or semantic - that cannot be incorporated into the gist of an experience (Brainerd et al., 2009). Finally, hippocampal pattern separation theories make a third prediction: that older adults should be impaired on any mnemonic discrimination involving a high degree of overlap between stimuli, including perceptual overlap at the level of single items (Bakker, Kirwan, Miller, \& Stark, 2008; Yassa et al., 2011). 
The R-H account shares several properties with these theories, for example, that deterioration of MTL structures is critical to age-related cognitive deficits, that compromised associative representations play an important role, and that older adults should exhibit reduced ability to resolve overlap between stimulus representations (Barense, Groen, et al., 2012; Cowell, 2012). However, the predictions of the R-H account for the effects of semantic and perceptual interference in aging differ from all three sets of predictions just outlined. In the case of perceptual interference, the R-H account predicts that, if the stimuli contain sufficient feature-overlap to necessitate conjunctive representations for their unique identification, old age should increase susceptibility to such interference. This prediction is similar to those of the hippocampal pattern separation account and Fuzzy Trace Theory, but differs from the Associative Deficit Hypothesis, which predicts no age-related susceptibility to perceptual interference unless the perceptual material is associatively related to the test discriminanda (in the present study, it was not). In the case of semantic interference, the $\mathrm{R}-\mathrm{H}$ account makes a unique prediction: that older adults should be paradoxically less affected by such interference than young adults, because they are less able to apprehend and encode the interfering associative relations between studied items. In this case, all three alternative theories appear to predict age-related impairments, because semantic interference (1) is associative (Associative Deficit Hypothesis), (2) necessitates memory for verbatim details in order to discriminate accurately between semantically-related targets and lures (Fuzzy Trace Theory), and (3) causes greater overlap between item representations (hippocampal pattern separation account). Thus, the present finding of an interaction between age and interference type, with older adults relatively shielded from semantic interference and impaired by perceptual interference compared to young adults, seems to be compatible with the predictions of the R-H account alone.

Our interpretation of the key finding is that older adults' pattern of susceptibility to interference is caused by compromised conjunctive representations in the MTL or nearby temporal lobe regions. Specifically, we propose that older adults possess deficits in conjunctive representations in both a phonemic/orthographic hierarchy of word representations, and in a separate semantic hierarchy. In the phonemic/orthographic hierarchy, the conjunctions that bind together the phonemic and/or orthographic features that uniquely identify a word are compromised, reducing the ability to correctly identify a target word as studied by discriminating it from unstudied words that share perceptual features. In the semantic hierarchy, the representations of inter-item semantic associations are compromised, reducing the ability to extract and encode the semantic associations that in healthy, young individuals impair the 
discriminability of targets from related lures at test. These compromised conjunctive representations thus have two opposing effects: they tend to increase older adults' susceptibility to interference from phonemically/orthographically-related items, but paradoxically shield older adults from interference caused by semantically-related items. This interpretation is paradoxical in that it explains a tendency for older adults to be facilitated relative to young adults in terms of a cognitive deficit: compromised associative-semantic representations protect mnemonic discrimination ability in the face of increasing semantic interference. A skeptic might reasonably argue, instead, that the present data are better explained by an age-related improvement in associative-semantic representations. However, this interpretation seems less plausible than our preferred interpretation, for two reasons. First, a number of studies have reported a decline in semantic memory function with normal aging (e.g., Bäckman \& Nilsson, 1996; Berkowitz, 1953; Kaufman, 2001; Lindenberger \& Baltes, 1994; Rönnlund et al., 2005). Indeed, even in theoretical accounts of memory in aging that posit the over-reliance of older adults on semantic gist, it is not assumed that older individuals encode semantic information more deeply than young adults (e.g., Koutstaal et al., 2003). Second, the data from our own study point to the same conclusion: comparing semantic discrimination performance in older and younger adults (by taking d' of related lures from targets and collapsing across category size) reveals that older adults performed significantly worse, $t(65.37)=-2.17, p=.034$. In other words, we found no evidence of superior ability to discriminate semantically related items in older adults, as would be expected if semantic representations become more precise and detailed with age. Importantly, it is also not the case that semantic discrimination in older adults was at floor - their d' scores from semantic categories, collapsed across category size, differed significantly from zero, t (35) = 16.98, p <.001 - ruling out the possibility that the shielding from semantic interference seen in this group occurred simply because performance had no room to go lower with additional interference.

A number of prior studies have examined the existence and separability of age-related deficits in perceptual and semantic memory. The results of these studies conflict with each other: one reported greater age-related susceptibility to semantic interference (Koutstaal et al., 2003); another revealed age-related deficits in perceptual rather than semantic mnemonic discrimination (Ly et al., 2013); and two further studies found that older adults are impaired by both perceptual and semantic interference (Budson et al., 2003; Pidgeon \& Morcom, 2014). Moreover, the results of the present study align with none of these prior reports. Even the findings from Ly et al. (2013), which appear to yield conclusions similar to our own, in fact differed critically from our results, as explained below. However, the heterogeneity of prior results is perhaps unsurprising in light of the 
considerable range of task parameters employed by these studies (e.g., category size, stimulus format, between- versus within-subject designs, and means of manipulating semantic and perceptual content), as well as the variety of dependent variables (e.g., d', FA rate, or baselinecorrected FA rate) that the authors used. In the next section, we explain two ways in which the task design and data analyses of the present study were optimized to test our a priori predictions and to rule out important confounds that may have applied to prior studies.

First, many DRM studies examining memory in older adults have focused principally on FA rates, which - unlike d' or A' measures derived using SDT - do not account for potential differences in response bias between groups (Budson et al., 2003; Koutstaal et al., 2003; Pidgeon \& Morcom, 2014). In some analyses of the data, critical lure FA rates were corrected for baseline levels of false recognition by subtracting the FA rate for unrelated lures (Budson et al., 2003; Koutstaal et al., 2003; Pidgeon \& Morcom, 2014). Although there may be some theoretical account of guessing (i.e., a psychological model that offers an explanation of how unrelated lures come to be endorsed as targets) under which subtraction of FA rate for unrelated lures is an appropriate correction, none was provided for these studies. In fact, this correction appears to rest upon an assumption that false memory and guessing are mutually exclusive; this seems implausible, given that one property of such a model is that as guess rate increases false memory must decrease. Thus, it is unclear how to appropriately interpret critical lure FA rates that have been corrected in this way, and it cannot be assumed that such a correction controls for response bias. In contrast, SDT is based upon a welldefined psychological model and provides a measure of mnemonic discriminability, d', that is uncontaminated by response bias. This increases confidence that the measure of performance provided by d' is a reliable index of mnemonic processing alone.

Second, some prior DRM studies have employed only one category size (Budson et al., 2003; Ly et al., 2013), or have employed more than one category size but have not placed great import on finding an interaction between category size and other key factors such as interference type and age group (Koutstaal et al., 2003; Pidgeon \& Morcom, 2014; but see Koutstaal \& Schacter, 1997). A manipulation of category size provides a manipulation of the amount of mnemonic interference from the study phase. As noted above, this manipulation is crucial in ruling out item confounds that may exist when stimuli cannot be counterbalanced between conditions (e.g., perceptual versus semantic categories). Thus, to demonstrate a differential effect of semantic versus perceptual interference unconfounded by inherent item properties, it is necessary to find an interaction of category size and interference type. To demonstrate a differential effect that differs by age, it is 
necessary to find a three-way interaction of category size, interference type and age group, as reported in this study for the first time.

The manipulation of category size is one way in which our study differed from that of Ly et al. (2013), and consideration of this factor reveals an important difference between the two sets of findings. Because Ly et al. tested only one category size, the most analogous comparison to their data that we can make is an assessment of $d$ ' for related lures collapsed across category size, which in fact reveals the opposite pattern of results from Ly et al.: a marginal interaction between Age Group and Interference Type, $F(1,110)=3.48, p=.065$, in which older adults were impaired by semantic (Welch's $t(65.37)=-2.17, \mathrm{p}=.034)$ but not phonemic/orthographic $(t(66.28)=-0.52, \mathrm{p}=$ .608) overlap between targets and lures (see Figure 4). In order to make the closest possible comparison to Ly et al., who used very small categories of 1 item, we also examined d' for 2-item categories alone, but again found the opposite pattern from Ly et al. (2013): an interaction between Interference Type and Age Group $(F(1,110)=9.565, p=.003)$ in which phonemic/orthographic d' scores did not differ by age ( $\mathrm{p}=.825)$ but semantic d' scores did ( $\mathrm{p}=.005)$. Thus, unlike in Ly et al., older adults in our study were not better at semantic discrimination and worse at perceptual discrimination per se, indeed, we found the opposite pattern. Two further differences between the two studies may have contributed to the discrepant results. First, Ly et al. used very small (1-item) categories and presented items such that interference occurred only at test: each related lure in the test list was related to one item from study. Second, the dependent variable for which Ly et al. reported the key interaction of age (young/old) and task (perceptual/semantic) was d' between targets and unrelated foils, ignoring hits and FAs for related lures. In combination with 1-item categories, this targets-versus-foils d' essentially provides a measure of recognition memory in the presence of perceptual or semantic similarity at the time of test. In contrast, participants in our study saw multiple items ( 2 or 8 ) from each category at study, and d' was calculated between targets and related lures, providing a measure of recognition memory that is influenced by studyrelated interference. Thus, the two experimental designs may have engendered quite different representational and cognitive demands (as discussed by Ly et al., 2013). We note that Ly et al. reported another dependent variable that did measure the discriminability of targets from related lures, but the age*task interaction for this measure was not significant, making it difficult to conclude in favor of a differential effect of perceptual versus semantic study-related interference in older compared to young adults.

In sum, in this study we set forth the predictions of the R-H account of cognition for memory in healthy aging, and tested those predictions with a recognition memory experiment employing 
the DRM paradigm. We found that older adults, relative to young adults, were paradoxically shielded from semantic interference but impaired by perceptual interference. This pattern of results appears to be at odds with most current theories of memory in aging, but is in line with the $\mathrm{R}-\mathrm{H}$ account of age-related memory loss, which explains the performance of older participants in terms of compromised conjunctive representations in anteromedial temporal lobe regions. 


\section{Acknowledgements}

This work was supported by the National Science Foundation Award \#1554871. The sponsors had no involvement in the execution or reporting of this work. 


\section{References}

Abrams, L., Trunk, D. L., \& Merrill, L. a. (2007). Why a superman cannot help a tsunami: activation of grammatical class influences resolution of young and older adults' tip-of-the-tongue states. Psychology and Aging, 22(4), 835-45. http://doi.org/10.1037/0882-7974.22.4.835

Bäckman, L., \& Nilsson, L.-G. (1996). Semantic memory functioning across the adult life span. European Psychologist, 1(1), 27-33. http://doi.org/10.1027/1016-9040.1.1.27

Bakker, A., Kirwan, C. B., Miller, M., \& Stark, C. E. L. (2008). Pattern Separation in the Human Hippocampal CA3 and Dentate Gyrus, (March), 1640-1643.

Barense, M. D., Bussey, T. J., Lee, A. C. H., Rogers, T. T., Davies, R. R., Saksida, L. M., ... Graham, K. S. (2005). Functional Specialization in the Human Medial Temporal Lobe. The Journal of Neuroscience, 25(44), 10239-10246. http://doi.org/10.1523/JNEUROSCI.2704-05.2005

Barense, M. D., Gaffan, D., \& Graham, K. S. (2007). The human medial temporal lobe processes online representations of complex objects. Neuropsychologia, 45(13), 2963-2974. http://doi.org/10.1016/j.neuropsychologia.2007.05.023

Barense, M. D., Groen, I. I. A., Lee, A. C. H., Yeung, L. K., Brady, S. M., Gregori, M., ... Henson, R. N. A. (2012). Intact memory for irrelevant information impairs perception in amnesia. Neuron, 75(1), 157-167. http://doi.org/10.1016/j.neuron.2012.05.014

Barense, M. D., Ngo, J. K. W., Hung, L. H. T., \& Peterson, M. A. (2012). Interactions of memory and perception in amnesia: The figure-ground perspective. Cerebral Cortex, 22(11), 2680-2691. http://doi.org/10.1093/cercor/bhr347

Barense, M. D., Rogers, T. T., Bussey, T. J., Saksida, L. M., \& Graham, K. S. (2010). Influence of conceptual knowledge on visual object discrimination: Insights from semantic dementia and MTL amnesia. Cerebral Cortex, 20(11), 2568-2582. http://doi.org/10.1093/cercor/bhq004

Bartko, S. J., Cowell, R. A., Winters, B. D., Bussey, T. J., \& Saksida, L. M. (2010). Heightened susceptibility to interference in an animal model of amnesia: Impairment in encoding, storage, retrieval - or all three? Neuropsychologia, 48, 2987-2997. http://doi.org/10.1016/j.neuropsychologia.2010.06.007

Bartko, S. J., Winters, B. D., Cowell, R. A., Saksida, L. M., \& Bussey, T. J. (2007a). Perceptual functions of perirhinal cortex in rats: zero-delay object recognition and simultaneous oddity discriminations. The Journal of Neuroscience, 27(10), 2548-2559. http://doi.org/10.1523/JNEUROSCI.5171-06.2007

Bartko, S. J., Winters, B. D., Cowell, R. A., Saksida, L. M., \& Bussey, T. J. (2007b). Perirhinal cortex resolves feature ambiguity in configural object recognition and perceptual oddity tasks. Learning \& Memory, 14, 821-832. http://doi.org/10.1101/lm.749207

Berkowitz, B. (1953). The Wechsler-Bellevue performance of white males past age 50. Journal of Gerontology, 8, 76-80.

Brainerd, C. J., Reyna, V. F., \& Howe, M. L. (2009). Trichotomous Processes in Early Memory Development, Aging, and Neurocognitive Impairment : A Unified Theory, 116(4), 783-832. http://doi.org/10.1037/a0016963

Bruffaerts, R., Dupont, P., Peeters, R., Deyne, S. De, Storms, G., \& Vandenberghe, R. (2013). Similarity of fMRI activity patterns in left perirhinal cortex reflects semantic similarity between words. The Journal of Neuroscience, 33(47), 18597-18607. http://doi.org/10.1523/JNEUROSCI.154813.2013

Budson, A. E., Sullivan, A. L., Daffner, K. R., \& Schacter, D. L. (2003). Semantic versus phonological false recognition in aging and Alzheimer's disease. Brain and Cognition, 51(3), 251-261. http://doi.org/10.1016/S0278-2626(03)00030-7

Bussey, T. J., \& Saksida, L. M. (2002). The organization of visual object representations: A connectionist model of effects of lesions in perirhinal cortex. The European Journal of Neuroscience, 15, 355-364. http://doi.org/10.1046/j.0953-816x.2001.01850.x 
Bussey, T. J., Saksida, L. M., \& Murray, E. A. (2002). Perirhinal cortex resolves feature ambiguity in complex visual discriminations. European Journal of Neuroscience, 15, 365-374. http://doi.org/10.1046/j.0953-816x.2001.01851.x

Campbell, K. L., Hasher, L., \& Thomas, R. C. (2010). Hyper-Binding. Psychological Science, 21(3), 399-405. http://doi.org/10.1177/0956797609359910

Chadwick, M. J., Anjum, R. S., Kumaran, D., Schacter, D. L., Spiers, H. J., \& Hassabis, D. (2016). Semantic representations in the temporal pole predict false memories. Proceedings of the National Academy of Sciences, 201610686. http://doi.org/10.1073/pnas.1610686113

Chalfonte, B. L., \& Johnson, M. K. (1996). Feature memory and binding in young and older adults. Memory \& Cognition, 24(4), 403-416. http://doi.org/10.3758/BF03200930

Clarke, A., \& Tyler, L. K. (2014). Object-specific semantic coding in human perirhinal cortex. Journal of Neuroscience, 34(14), 4766-4775. http://doi.org/10.1523/JNEUROSCI.2828-13.2014

Cohen, L., \& Dehaene, S. (2004). Specialization within the ventral stream: The case for the visual word form area. NeuroImage, 22(1), 466-476. http://doi.org/10.1016/j.neuroimage.2003.12.049

Cohen, N. J., Ryan, J., Hunt, C., Romine, L., Wszalek, T., \& Nash, C. (1999). Hippocampal system and declarative (relational) memory: Summarizing the data from functional neuroimaging studies. Hippocampus, 9, 83-98. http://doi.org/10.1002/(SICI)1098-1063(1999)9:1<83::AIDHIPO9>3.0.CO;2-7

Coltheart, M., Rastle, K., Perry, C., Langdon, R., \& Ziegler, J. (2001). DRC: A dual route cascaded model of visual word recognition and reading aloud. Psychological Review, 108(1), 204-256. http://doi.org/10.1037/0033-295X.108.1.204

Cowell, R. A. (2012). Computational models of perirhinal cortex function. Hippocampus, 22(10), 1952-1964. http://doi.org/10.1002/hipo.22064

Cowell, R. A., Bussey, T. J., \& Saksida, L. M. (2006). Why does brain damage impair memory? A connectionist model of object recognition memory in perirhinal cortex. The Journal of Neuroscience, 26(47), 12186-12197. http://doi.org/10.1523/JNEUROSCI.2818-06.2006

Crum, R. M., Anthony, J. C., Bassett, S. S., \& Folstein, M. F. (1993). Population-based norms for the Mini-Mental State Examination by age and educational level. JAMA, 269(18), 2386-2391.

Deese, J. (1959). On the prediction of occurrence of particular verbal intrusions in immediate recall. Journal of Experimental Psychology, 58(1), 17-22. http://doi.org/10.1037/h0046671

Dell, G. S. (1986). A spreading-activation theory of retrieval in sentence production. Psychological Review, 93(3), 283-321.

Devitt, A. L., \& Schacter, D. L. (2016). False memories with age: Neural and cognitive underpinnings. Neuropsychologia, 91, 346-359. http://doi.org/10.1016/j.neuropsychologia.2016.08.030

Dewhurst, S. A., Hitch, G., \& Barry, C. (1998). Separate effects of word frequency and age of acquisition in recogntion and recall. Journal of Experimental Psychology, 24(2), 284-298.

Duarte, A., Henson, R. N., \& Graham, K. S. (2008). The effects of aging on the neural correlates of subjective and objective recollection. Cerebral Cortex, 18(9), 2169-2180. http://doi.org/10.1093/cercor/bhm243

Eichenbaum, H., Otto, T., \& Cohen, N. J. (1994). Two functional components of the hippocampal memory system. Behavioral and Brain Sciences, 17(3), 449-472. http://doi.org/10.1017/S0140525X00035391

Folstein, M. F., Folstein, S. E., \& McHugh, P. R. (1975). “Mini-mental state”: A practical method for grading the cognitive state of patients for the clinician. Journal of Psychiatric Research, 12(3), 189-198.

Gallo, D. A. (2006). Associative illusions of memory: False memory research in DRM and related tasks. New York, NY: Psychology Press.

Giovanello, K. S., Schnyer, D. M., \& Verfaellie, M. (2004). A critical role of the anterior hippocampus in relational memory: Evidence from an fMRI study comparing associative and item 
recognition. Hippocampus, 14, 5-8. http://doi.org/10.1002/hipo.10182

Glezer, L. S., Kim, J., Rule, J., Jiang, X., \& Riesenhuber, M. (2015). Adding words to the brain's visual dictionary: Novel word learning selectively sharpens orthographic representations in the VWFA. Journal of Neuroscience, 35(12), 4965-4972. http://doi.org/10.1523/JNEUROSCI.403114.2015

Gordon, J. K., \& Kurczek, J. C. (2013). The ageing neighbourhood: phonological density in naming. Language and Cognitive Processes, $0(0), 1-19$. http://doi.org/10.1080/01690965.2013.837495

Hautus, M. J. (1995). Corrections for extreme proportions and their biasing effects on estimated values of d'. Behavior Research Methods, Instruments, \& Computers, 27(1), 46-51. http://doi.org/10.3758/BF03203619

Hickok, G., \& Poeppel, D. (2007). The cortical organization of speech processing. Nature Reviews. Neuroscience, 8(5), 393-402.

Holden, H. M., Hoebel, C., Loftis, K., \& Gilbert, P. E. (2012). Spatial Pattern Separation in Cognitively Normal Young and Older Adults, 1832, 1826-1832. http://doi.org/10.1002/hipo.22017

Kaufman, A. S. (2001). WAIS-III IQs, Horn's theory, and generational changes from young adulthood to old age. Intelligence, 29, 131-167. http://doi.org/10.1016/S0160-2896(00)00046-5

Kinoshita, S. (1995). The Word-Frequency Effect In Recognition Memory Versus Repetition Priming. Memory \& Cognition, 23(5), 569-580. http://doi.org/10.3758/bf03197259

Koutstaal, W., Reddy, C., Jackson, E. M., Prince, S., Cendan, D. L., \& Schacter, D. L. (2003). False recognition of abstract versus common objects in older and younger adults: testing the semantic categorization account. Journal of Experimental Psychology. Learning, Memory, and Cognition, 29(4), 499-510. http://doi.org/10.1037/0278-7393.29.4.499

Koutstaal, W., \& Schacter, D. L. (1997). Gist-Based False Recognition of Pictures in Older and Younger Adults. Journal of Memory and Language, 37, 555-583. http://doi.org/10.1006/jmla.1997.2529

Lawrence, M. A. (2015). ez: Easy Analysis and Visualization of Factorial Experiments. Retrieved from https://cran.r-project.org/package $=\mathrm{ez}$

Lee, A. C. H. (2006). Differentiating the Roles of the Hippocampus and Perirhinal Cortex in Processes beyond Long-Term Declarative Memory: A Double Dissociation in Dementia. Journal of Neuroscience, 26(19), 5198-5203. http://doi.org/10.1523/JNEUROSCI.3157-05.2006

Lee, A. C. H., Bandelow, S., Schwarzbauer, C., Henson, R. N. A., \& Graham, K. S. (2006). Perirhinal cortex activity during visual object discrimination: An event-related fMRI study. NeuroImage, 33, 362-373. http://doi.org/10.1016/j.neuroimage.2006.06.021

Lee, A. C. H., Buckley, M. J., Pegman, S. J., Spiers, H., Scahill, V. L., Gaffan, D., ... Graham, K. S. (2005). Specialization in the medial temporal lobe for processing of objects and scenes. Hippocampus, 15, 782-797. http://doi.org/10.1002/hipo.20101

Lee, A. C. H., Bussey, T. J., Murray, E. A., Saksida, L. M., Epstein, R. A., Kapur, N., ... Graham, K. S. (2005). Perceptual deficits in amnesia: Challenging the medial temporal lobe "mnemonic" view. Neuropsychologia, 43(1), 1-11. http://doi.org/10.1016/j.neuropsychologia.2004.07.017

Lee, A. C. H., Levi, N., Davies, R. R., Hodges, J. R., \& Graham, K. S. (2007). Differing profiles of face and scene discrimination deficits in semantic dementia and Alzheimer's disease. Neuropsychologia, 45(9), 2135-2146. http://doi.org/10.1016/j.neuropsychologia.2007.01.010

Lee, A. C. H., Scahill, V. L., \& Graham, K. S. (2008). Activating the medial temporal lobe during oddity judgment for faces and scenes. Cerebral Cortex, 18, 683-696. http://doi.org/10.1093/cercor/bhm104

Lezak, M. D., Howieson, D. B., \& Loring, D. W. (2004). Neuropsychological Assessment (4th ed.). New York, NY: Oxford University Press.

Lindenberger, U., \& Baltes, P. B. (1994). Sensory functioning and intelligence in old age: A strong connection. Psychology and Aging, 9(3), 339-355. http://doi.org/10.1037/0882-7974.9.3.339 
Ly, M., Murray, E., \& Yassa, M. A. (2013). Perceptual versus conceptual interference and pattern separation of verbal stimuli in young and older adults. Hippocampus, 23(6), 425-430. http://doi.org/10.1002/hipo.22110

Macmillan, N. A., \& Creelman, C. D. (2005). Detection Theory: A User's Guide (2nd ed.). Mahwah, NJ: Lawrence Erlbaum Associates.

Madigan, S., \& Neuse, J. (2004). False recognition and word length: A reanalysis of Roediger, Watson, McDermott, and Gallo (2001) and some new data. Psychonomic Bulletin \& Review, 11(3), 567-573. http://doi.org/10.3758/BF03196612

Manns, J. R., Hopkins, R. O., \& Squire, L. R. (2003). Semantic memory and the human hippocampus. Neuron, 38(1), 127-133. http://doi.org/10.1016/S0896-6273(03)00146-6

McTighe, S. M., Cowell, R. A., Winters, B. D., Bussey, T. J., \& Saksida, L. M. (2010). Paradoxical false memory for objects after brain damage. Science, 330, 1408-1410. http://doi.org/10.1126/science.1194780

Naveh-Benjamin, M. (2000). Adult age differences in memory performance: Tests of an associative deficit hypothesis. Journal of Experimental Psychology: Learning, Memory, and Cognition, 26(5), 1170-1187. http://doi.org/10.1037//0278-7393.26.5.1170

Naveh-Benjamin, M., Hussain, Z., Guez, J., \& Bar-On, M. (2003). Adult age differences in episodic memory: Further support for an associative-deficit hypothesis. Journal of Experimental Psychology: Learning, Memory, and Cognition, 29(5), 826-837. http://doi.org/10.1037/02787393.29.5.826

Newman, M. C., \& Kaszniak, A. W. (2000). Spatial memory and aging: Performance on a human analog of the Morris water maze. Aging, Neuropsychology, and Cognition, 7(2), 86-93. http://doi.org/10.1076/1382-5585(200006)7:2;1-U;FT086

Pidgeon, L. M., \& Morcom, A. M. (2014). Age-related increases in false recognition: The role of perceptual and conceptual similarity. Frontiers in Aging Neuroscience, 6(OCT), 1-17. http://doi.org/10.3389/fnagi.2014.00283

Raz, N., Gunning-Dixon, F. M., Head, D., Dupuis, J. H., \& Acker, J. D. (1998). Neuroanatomical correlates of cognitive aging: Evidence from structural magnetic resonance imaging. Neuropsychology, 12(1), 95-114. http://doi.org/10.1037//0894-4105.12.1.95

Raz, N., Rodrigue, K. M., Head, D., Kennedy, K. M., \& Acker, J. D. (2004). Differential aging of the medial temporal lobe: A study of a five-year change. Neurology, 62, 433-438. http://doi.org/10.1212/01.WNL.0000106466.09835.46

Roediger III, H. L., \& McDermott, K. B. (1995). Creating false memories: Remembering words not presented in lists. Journal of Experimental Psychology: Learning, Memory, and Cognition, 21(4), 803-814. http://doi.org/10.1037/0278-7393.21.4.803

Roediger III, H. L., Watson, J. M., McDermott, K. B., \& Gallo, D. A. (2001). Factors that determine false recall: a multiple regression analysis. Psychonomic Bulletin \& Review, 8(3), 385-407. http://doi.org/10.3758/BF03196177

Rönnlund, M., Nyberg, L., Bäckman, L., \& Nilsson, L.-G. (2005). Stability, growth, and decline in adult life span development of declarative memory: Cross-sectional and longitudinal data from a population-based study. Psychology and Aging, 20(1), 3-18. http://doi.org/10.1037/08827974.20.1.3

Sadil, P. S., \& Cowell, R. A. (2017). A Computational Model of Perceptual and Mnemonic Deficits in Medial Temporal Lobe Amnesia. Journal of Cognitive Neuroscience, 29(6), 1075-1088. http://doi.org/10.1162/jocn

Schacter, D. L., Kaszniak, A. W., Kihlstrom, J. F., \& Valdiserri, M. (1991). The relation between source memory and aging. Psychology and Aging, 6(4), 559-568. http://doi.org/10.1037/08827974.6.4.559

Schacter, D. L., Koutstaal, W., \& Norman, K. A. (1997). False memories and aging. Trends in Cognitive Sciences, 1(6), 229-236. 
Schacter, D. L., Osowiecki, D., Kaszniak, A. W., Kihlstrom, J. F., \& Valdiserri, M. (1994). Source memory: extending the boundaries of age-related deficits. Psychology and Aging, 9(1), 81-89. http://doi.org/10.1037/0882-7974.9.1.81

Schaie, K. W. (2005). What can we learn from longitudinal studies of adult development? Research in Human Development, 2(3), 133-158. http://doi.org/http://dx.doi.org/10.1207/s15427617rhd0203_4

Scheerer, N., \& Marrone, D. F. (2014). Age-Related Deficits in Conjunctive Representation of Complex Objects, 214-219. http://doi.org/10.2174/1874609808666150201215549

Scoville, W., \& Milner, B. (1957). Loss of recent memory after bilateral hippocampal lesions. J Neurol Neurosurg Psychiatry, 20, 11-21.

Sherman, S. M., \& Jordan, T. R. (2011). Word-frequency effects in long-term semantic priming and false memory. British Journal of Psychology, 102(3), 559-568. http://doi.org/10.1111/j.20448295.2011.02017.x

Shiffrin, R., Huber, D. E., \& Marinelli, K. (1995). Effects of Categoy Length and Strength on Familiarity Recognition. Journal of Experimental Psychology: Learning, Memory, and Cognition, 21(2), 267-287.

Silver, H., Goodman, C., \& Bilker, W. B. (2012). Impairment in associative memory in healthy aging is distinct from that in other types of episodic memory. Psychiatry Research, 197(1-2), 135-139. http://doi.org/10.1016/j.psychres.2012.01.025

Snodgrass, J. G., \& Corwin, J. (1988). Pragmatics of measuring recognition memory: applications to dementia and amnesia. Journal of Experimental Psychology. General, 117(1), 34-50. http://doi.org/10.1037/0096-3445.117.1.34

Spencer, W. D., \& Raz, N. (1995). Differential effects of aging on memory for content and context: A meta-analysis. Psychology and Aging, 10(4), 527-539. http://doi.org/10.1037/08827974.10.4.527

Squire, L. R., \& Wixted, J. T. (2011). The Cognitive Neuroscience of Human Memory Since H.M. Annual Review of Neuroscience, 34(1), 259-288. http://doi.org/10.1146/annurev-neuro061010-113720

Squire, L. R., \& Zola-morgan, S. (1991). The Medial Temporal Lobe Memory System Human Memory. Science, 253(5026), 1380-1386. http://doi.org/10.1146/annurev.neuro.27.070203.144130

Stark, S. M., Yassa, M. A., \& Stark, C. E. L. (2010). Individual differences in spatial pattern separation performance associated with healthy aging in humans. Learning \& Memory, 17(6), 284-288. http://doi.org/10.1101/lm.1768110

Team, R. C. (2016). R: A language and environment for statistical computing. Vienna, Austria: R Foundation for Statistical Computing. Retrieved from https://www.r-project.org/

Tombaugh, T. N. (2004). Trail Making Test A and B : Normative data stratified by age and education. Archives of Clinical Neuropsychology, 19, 203-214. http://doi.org/10.1016/S08876177(03)00039-8

Wang, D., Chalk, J. B., Rose, S. E., de Zubicaray, G., Cowin, G., Galloway, G. J., ... Semple, J. (2002). MR image-based measurement of rates of change in volumes of brain structures. Part II: Application to a study of Alzheimer's disease and normal aging. Magnetic Resonance Imaging, 20, 41-48. http://doi.org/10.1016/S0730-725X(02)00472-1

Warrington, E. K., \& James, M. (1991). The Visual Object and Space Perception battery. Bury St. Edmunds, UK: Thames Valley Company.

Warrington, E. K., \& Shallice, T. (1984). Category specific semantic impairments. Brain, 107(3), 829853. http://doi.org/10.1093/brain/107.3.829

Wechsler, D. (2008). Wechsler Adult Intelligence Scale-Fourth Edition. San Antonio, TX: Pearson.

Wechsler, D. (2009). Wechsler Memory Scale-Fourth Edition. San Antonio, TX: Pearson.

Yassa, M. A., Lacy, J. W., Stark, S. M., Albert, M. S., Gallagher, M., \& Stark, C. E. L. (2011). Pattern Separation Deficits Associated With Increased Hippocampal CA3 and Dentate Gyrus Activity in 
Nondemented Older Adults. Hippocampus, 21, 968-979. http://doi.org/10.1002/hipo.20808 Yassa, M. A., \& Stark, C. E. L. (2011). Pattern separation in the hippocampus. Trends in Neurosciences, 34(10), 515-525. http://doi.org/10.1016/j.tins.2011.06.006

Yeung, L.-K., Ryan, J. D., Cowell, R. A., \& Barense, M. D. (2013). Recognition memory impairments caused by false recognition of novel objects. Journal of Experimental Psychology: General, 142(4), 1384-1397. http://doi.org/10.1037/a0034021 
Appendix A

Semantic Word Categories

\begin{tabular}{|c|c|c|c|c|}
\hline Astronaut & Butterfly & Castle & Comedian & Diamond \\
\hline Atmosphere & Camouflage & Chateau & Buffon & Brilliance \\
\hline Cosmonaut & Caterpillar & Courtyard & Clown & Carat \\
\hline Gravity & Cocoon & Dungeon & Comic & Emerald \\
\hline Orbiting & Dragonfly & Feudal & Humorist & Glittering \\
\hline Rocket & Flutter & Fortress & Improvisation & Hardness \\
\hline Satellite & Fragile & Mansion & Joker & Precious \\
\hline Shuttle & Metamorphosis & Medieval & Lampoon & Priceless \\
\hline Spaceman & Monarch & Stronghold & Monologue & Rhinestones \\
\hline Voyager & Slight & Throne & Punster & Rubies \\
\hline Weightlessness & Wings & Towers & Slapstick & Sparkle \\
\hline Dinosaur & Fitness & Gambler & $\underline{\text { Infant }}$ & Lunatic \\
\hline Amphibians & Aerobics & Bettor & Babble & $\overline{\text { Asylum }}$ \\
\hline Artifacts & Barbells & Blackjack & Cradle & Demented \\
\hline Brontosaurus & Biceps & Bookie & Diapers & Deranged \\
\hline Extinction & Exertion & Casino & Highchair & Hallucinations \\
\hline Fossils & Jogging & Jackpot & Lullaby & Insanity \\
\hline Glaciers & Nutrition & Lottery & Pacifier & Madman \\
\hline Mammoth & Physique & Poker & Rattle & Manic \\
\hline Reptiles & Sweating & Roulette & Stork & Psychopath \\
\hline Skeletons & Toning & Stakes & Stroller & Psychotic \\
\hline Swamps & Workout & Wager & Teething & Ranting \\
\hline Magician & Phantom & Pyramid & Robbery & Tornado \\
\hline Conjure & $\overline{\text { Apparition }}$ & Catacombs & $\overrightarrow{\text { Assailant }}$ & Cyclone \\
\hline Enchanted & Beckon & Egyptian & Bandit & Funnel \\
\hline Hypnotist & Ghost & Embalming & Booty & Gusts \\
\hline Juggling & Ghoul & Hieroglyphics & Burglary & Sirens \\
\hline Rabbit & Gloomy & Mummies & Holdup & Spiral \\
\hline Sorcerer & Goblin & Pharaoh & Mugging & Twister \\
\hline Spells & Haunting & Tombs & Stealing & Typhoon \\
\hline Trickster & Paranormal & Triangular & Stickup & Whirling \\
\hline Vanish & Specter & Underworld & Theft & Whirlwind \\
\hline Wizard & Spooky & Vault & Wallet & Windstorm \\
\hline
\end{tabular}


Appendix B

Phonemic/Orthographic Word Categories

$\begin{array}{lllll}\text { Boon } & \text { Bun } & \text { Cat } & \text { Cop } & \text { Fate } \\ \text { Boom } & \text { Bud } & \text { Bat } & \text { Bop } & \text { Date } \\ \text { Boos } & \text { Bum } & \text { Cab } & \text { Cob } & \text { Face } \\ \text { Boot } & \text { Bus } & \text { Cam } & \text { Cod } & \text { Fade } \\ \text { Coon } & \text { But } & \text { Cap } & \text { Cog } & \text { Fake } \\ \text { Goon } & \text { Fun } & \text { Fat } & \text { Con } & \text { Fame } \\ \text { Loon } & \text { Gun } & \text { Hat } & \text { Cot } & \text { Gate } \\ \text { Moon } & \text { Nun } & \text { Mat } & \text { Hop } & \text { Hate } \\ \text { Noon } & \text { Pun } & \text { Pat } & \text { Mop } & \text { Late } \\ \text { Soon } & \text { Run } & \text { Rat } & \text { Pop } & \text { Mate } \\ \text { Toon } & \text { Sun } & \text { Sat } & \text { Top } & \text { Rate } \\ & & & & \\ \text { Mire } & \text { Role } & \text { Sip } & \text { Teal } & \text { West } \\ \text { Dire } & \text { Dole } & \text { Dip } & \text { Deal } & \text { Best } \\ \text { Fire } & \text { Hole } & \text { Hip } & \text { Heal } & \text { Lest } \\ \text { Hire } & \text { Mole } & \text { Lip } & \text { Meal } & \text { Nest } \\ \text { Mice } & \text { Pole } & \text { Nip } & \text { Peal } & \text { Pest } \\ \text { Mike } & \text { Robe } & \text { Rip } & \text { Real } & \text { Rest } \\ \text { Mile } & \text { Rode } & \text { Sin } & \text { Seal } & \text { Test } \\ \text { Mime } & \text { Rope } & \text { Sis } & \text { Team } & \text { Vest } \\ \text { Mine } & \text { Rose } & \text { Sit } & \text { Teas } & \text { Welt } \\ \text { Tire } & \text { Rote } & \text { Six } & \text { Teat } & \text { Went } \\ \text { Wire } & \text { Sole } & \text { Tip } & \text { Veal } & \text { Wept } \\ & & & & \end{array}$


Appendix C

Extra Words

\section{Semantic}

Antiquity

Apartment

Attic

Bagel

Biologist

Bison

Canvas

Carport

Convenience

Dachshund

Gazette

Gutter

Honeycomb

Housecoat

Industry

Inferno

Invitation

Jargon

Jasmine

Linguistics

Mechanical

Monoxide
Mooring

Opossum

Pauper

Piccolo

Podium

Promenade

Purple

Sable

Schoolyard

Scissors

Silhouette

Stationer

Storeroom

Synopsis

Thesaurus

Thicket

Tortilla

Tribesman

Triplicate

Undergrowth

Unformed

Warmhearted
Phonemic/Orthographic

Bird

Book

Bout

Bur

Coin

Cow

Foil

Fur

Joy

Lawn

Loud

Now

Null

Perk

Pull

Raw

Saw

Toy

Wood

Yaw 